\title{
Monitoreo del tratamiento de la osteoporosis con densitometria mineral ósea: cuándo cambiar de droga?
}

Steven R Cummings, Lisa Palermo, Warren Browner, et al. Monitoring osteoporosis therapy with bone densitometry. JAMA 2000; 283:1318-1321.

\section{Objetivo}

Evaluar si las pacientes que disminuyen la densidad mineral ósea (DMO) después de un año de tratamiento para la osteoporosis (OP) continúan perdiendo DMO al año siguiente.

\section{Diseño}

Es un estudio secundario (descriptivo) que surge de la revisión de dos trials randomizados doble ciego multicéntricos para el tratamiento de la osteoporosis ( The fracture intervention trial -FIT- y The multiple outcomes of raloxifene evaluation trial-MORE-).

\section{Lugar}

Departamento de epidemiología, bioestadística y medicina de la Universidad de California, San Francisco, USA.

\section{Pacientes}

El estudio FIT incluyó mujeres posmenopaúsicas (2634) con osteoporosis que no hubiesen recibido tratamiento para OP en los últimos 6 meses previos, randomizadas a recibir $5 \mathrm{mg} . / \mathrm{d}$ de alendronato o placebo.

El MORE trial incluyó mujeres postmenopaúsicas (7705) con osteoporosis que no hubiesen recibido bifosfonatos o estrógenos en los últimos 6 meses, randomizadas a recibir raloxifeno o placebo.

\section{Descripción de los test}

En el FIT midieron DMO de columna lumbar y de cadera al inicio, y luego de manera anual.; en el MORE también midieron DMO al inicio, a los 12 y a los 24 meses. En el primero se revisaba una mues- tra al azar de las DMO, en el segundo trabajo una central realizaba la corrección de los factores que pudiesen provocar cambios o diferencias en la performance de los densitómetros.

\section{Medición de resultados}

Densitometría mineral ósea de columna lumbar y de cadera basal, al año y a los dos años.

\section{Resultados}

En el FIT, las mujeres que recibieron alendronato y que durante el primer año habían mejorado su DMO en más de un $8 \%$ de incremento (IC $95 \% 9,7-11,2$ ), en el segundo año perdían un $1 \%$ (IC $95 \%-1,9-0,1)$; ocurriendo lo inverso cuando perdían DM0 en el primer año aumentaba en el segundo con un promedio de incremento del 4,7\%.

En el MORE trial se observaron resultados con la misma tendencia; cuando en el primer año se demostraba una pérdida mayor al $4 \%$, aumentaba en el segundo año con un promedio del $4,8 \%$.

\section{Conclusiones}

En este trabajo se observa que en las pacientes con osteoporosis que están en tratamiento, cuando su DMO no mejora o aún demuestra disminución probablemente mejoren su DMO al año siguiente si contínuan con igual fármaco. Estos resultados sugieren no modificar tratamientos efectivos para OP con un resultado desfavorable de la DMO en el primer año. Los autores plantean estos resultados como un ejemplo del concepto de regresión a la media.

\section{COMENTARIO}

En la última década han surgido numerosos estudios sobre prevención y tratamiento de osteoporosis, con diferentes alternativas farmacológicas, tiempos de seguimiento y con diferentes outcomes. Algunos miden marcadores bioquímicos, otros número de fracturas, pero la gran mayoría basan sus resultados en cambios de la densidad mineral ósea (DM0). En una revisión de la evidencia disponible en osteoporosis ${ }^{1}$, se plantea que al evaluar los diferentes tratamientos el efecto de la intervención se mide en gral a través de la DM0 asumiendo que su mejoría disminuye la chance de fracturas. No obstante, reconociendo que tampoco se dispone en la actualidad de un método mejor, el autor remarca que la DMO es un método indirecto de evaluación. En este punto hay estudios longitudinales que demostraron este tipo de relación, y otros que no observaron una buena correlación entre modificación de la DMO y riesgo de fracturas, por lo cual actualmente esta correlación no está claramente demostrada. Por otro lado tampoco se conocen bien las características del test como su sensibilidad, especifidad, reproducibilidad, etc. En la actualidad, en OP nos enfrentamos a varios interrogantes tales como: cuál es el tratamiento más costo-efectivo, qué significa (medido en riesgo de fractura) un incremento del $4 \%$ en la DMO? Y una disminución del 2\%?, cuál es el mejor método para monitoreo del tratamiento?, cuándo cambiar de droga? Estas dos últimas preguntas se replantean en este trabajo, que si bien es un estudio secundario los autores ilustran un fenómeno muy frecuente pero pocas veces reconocido: "la regresión a la media" y en este caso en particular sugiere no apresurarse en modificar un tratamiento probablemente efectivo para la OP. Por el momento surgen hipótesis y la necesidad de continuar estudiando esta entidad ya que por su prevalencia y repercusión tiene un gran impacto en la salud de la mujer.

Dra. Vilda Discacciati Unidad de Medicina Familiar y Preventiva. Hospital Italiano de Buenos Aires. 